\title{
Changes in Managed Care Activity in Outpatient Substance Abuse Treatment Organizations, 1995-2000
}

\author{
Jeffrey A. Alexander, PhD \\ Christy Harris Lemak, PhD \\ Cynthia I. Campbell, PhD
}

\begin{abstract}
Using nationally representative data from 1995 and 2000, this study examines trends in managed care penetration and activity among outpatient drug treatment organizations in the United States. Further, it investigates how managed care activity varies across different types of treatment providers and for public and private managed care programs. Overall, managed care activity has increased, with a greater proportion of units having managed care arrangements and a larger percentage of clients covered by managed care. In general, public managed care activity has increased and private managed care activity has decreased. Treatment providers report that they have fewer managed care arrangements, which may reflect consolidation in the managed behavioral care sector. Finally, growth in managed care among outpatient substance abuse treatment units affiliated with hospitals and mental health centers may signal a preference for providers that can effectively link substance abuse treatment with medical and social service provision, or, alternatively, that linkages with such organizations may provide the size necessary to assume the risks associated with managed care contracts.
\end{abstract}

\section{Introduction}

Managed care has made significant inroads into the substance abuse treatment sector. Specifically, enrollment in managed behavioral programs increased $155 \%$ from 1993 to 2000 , and $68 \%$ of all Americans with health insurance are now enrolled in some type of managed behavioral program. ${ }^{1}$ In 1999, 42 states offered managed behavioral programs for enrollees in Medicaid and other programs-a 3 -fold increase since $1996 .{ }^{2}$ Clearly, the managed behavioral care industry has experienced rapid growth and dramatic change in recent years. ${ }^{3-8}$ Little is known, however, about how these

Address correspondence to Christy Harris Lemak, PhD, Assistant Professor, Department of Health Services Administration, The University of Florida, PO Box 100195, Gainesville, FL 32610-0195. E-mail: clemak@hp.uft.edu.

Jeffrey A. Alexander, PhD, is Richard Carl Jelinek Professor of Health Management and Policy in the Department of Health Management and Policy in the School of Public Health at the University of Michigan, Ann Arbor, Mich.

Cynthia I. Campbell, PhD, is a Postdoctoral Fellow in the Department of Psychiatry at the University of California, San Francisco, Calif.

Journal of Behavioral Health Services \& Research, 2003, 30(4), 369-381. (C) 2003 National Council for Community Behavioral Healthcare. 
sector-wise trends in managed care have had an impact on organizational providers of substance abuse treatment.

As policymakers are enjoined to debate and evaluate the relative merits and disadvantages of managed behavioral care for the substance abuse sector, they must have a basic and accurate understanding of the extent to which managed care has penetrated the substance abuse treatment delivery system. While substance abuse treatment services are provided in a variety of settings, specialized outpatient facilities have become the predominant form of delivery, accounting for nearly 1 million clients in treatment each day. ${ }^{9}$ A key question, then, is how has managed behavioral care affected outpatient treatment providers, for it is at this level that both the positive and negative consequences of managed care will potentially occur. More specifically, what are the trends in managed care activity among outpatient treatment providers during this period of rapid and dramatic change?

The question is complex because drug abuse treatment providers operate in an extremely fragmented financing environment. Providers face a myriad of different types of funding, including federal, state, and private sources, each with potentially different eligibility requirements and payment mechanisms. ${ }^{10}$ Similarly, these sources may all incorporate, to a varying degree, what might be considered "managed care" practices. ${ }^{11,12}$ Adding further complexity to the issue of managed care activity among substance abuse providers is the possibility that managed care is unequally represented among different types of provider organizations. For example, private for-profit units may be more likely than public units to have higher levels of managed care because they are able to target clients covered by both private and public managed care plans.

Finally, managed care arrangements themselves vary widely in their organizational structure and operating techniques. Although Medicaid and private managed behavioral programs are considered the most common "types" of managed care programs, some treatment providers may have a significant involvement with other forms of managed care, including arrangements with employee assistance plans, direct contracts with self-insured firms, and service contracts with state substance abuse agencies.

The study addresses the following research questions. First, what are recent trends in managed care penetration and activity for the outpatient drug treatment sector in the United States? Second, how does managed care activity vary across different types of treatment providers and for public and private managed care programs? New longitudinal data are presented from the fourth (1995) and fifth (1999/2000) waves of the National Drug Abuse Treatment System Survey (NDATSS), a nationally representative study of outpatient substance abuse treatment (OSAT) providers. This investigation examines trends in managed care penetration and managed care activities for the outpatient drug abuse treatment sector overall and for different types of treatment organizations, including trends by provider ownership type (private not-for-profit, private for-profit, or public), affiliation (hospital, mental health center, or freestanding), size, urbanicity, and region. To illustrate relevant trends and statistics, data are first presented from nationally representative samples of organizations for 1995 and 2000. Next, trends are examined among those treatment organizations that participated in managed care during these time periods.

\section{Methods}

The NDATSS is a national study of OSAT units conducted by the University of Michigan's Institute for Social Research (ISR). The study began in 1984, and has completed 5 waves of data collection. This article presents longitudinal data from the NDATSS, using the fourth and fifth waves since they included questions on managed care activity. The fourth wave was conducted in 1995, and the fifth wave in 1999/2000.

The details of the study design and instruments used have been described elsewhere. ${ }^{13}$ Briefly, the NDATSS uses a mixed panel design, which combines elements from panel and cross-sectional designs. Data are collected from the same national sample of outpatient drug abuse treatment units 
that have been sampled and screened as part of prior waves of the study. These panel units are combined with a new group of randomly selected OSAT units to ensure that the sample remains nationally representative. The new units are selected for participation from a sampling frame of the most complete list of the nation's OSAT units, compiled by ISR. ${ }^{14,15}$ In the fourth wave, the sample was stratified by ownership type, treatment modality (methadone or non-methadone), and organizational affiliation. The Wave 5 sample was stratified by treatment modality (methadone or non-methadone).

The NDATSS is a telephone survey of the administrative director and clinical supervisor at each OSAT unit. After screening and nonresponse, 618 organizations completed interviews in Wave 4 and 745 organizations completed interviews in Wave 5, with response rates of $88 \%$ and $89 \%$ respectively. In both waves, each participating organization was assigned a weight that accounted for probability of entry into the study and for nonresponse. ${ }^{14,15}$ The weights were applied to the 2 waves to make the data nationally representative. Table 1 displays the weighted and unweighted 1995 and 2000 samples by ownership type, affiliation, size, urbanicity, and region. In addition, total number of clients was used to weight the percentage of clients in managed care variables in Tables 2, 5, and 6, and total revenue was used to weight the percentage of revenue from managed care variables in Tables 5 and 6.

Table 1

Characteristics of 2 national samples of outpatient substance abuse treatment units, 1995 and 2000, by control status, unit affiliation, unit size, urbanicity, and region

\begin{tabular}{|c|c|c|c|c|c|c|}
\hline & \multicolumn{2}{|c|}{ Unweighted } & \multicolumn{2}{|c|}{ Weighted ${ }^{*}$} & \multicolumn{2}{|c|}{ \# of units } \\
\hline & 1995 & 2000 & 1995 & 2000 & 1995 & 2000 \\
\hline \multicolumn{7}{|l|}{ Ownership status } \\
\hline Private for profit & $14 \%$ & $24 \%$ & $17 \%$ & $19 \%$ & 85 & 176 \\
\hline Private not-for-profit & $62 \%$ & $57 \%$ & $52 \%$ & $54 \%$ & 380 & 424 \\
\hline Public & $24 \%$ & $19 \%$ & $31 \%$ & $27 \%$ & 146 & 144 \\
\hline \multicolumn{7}{|l|}{ Affiliation } \\
\hline Freestanding & $26 \%$ & $33 \%$ & $25 \%$ & $31 \%$ & 161 & 242 \\
\hline Hospital & $19 \%$ & $13 \%$ & $17 \%$ & $13 \%$ & 115 & 94 \\
\hline Mental health center & $22 \%$ & $15 \%$ & $27 \%$ & $18 \%$ & 138 & 110 \\
\hline Other & $32 \%$ & $38 \%$ & $29 \%$ & $37 \%$ & 195 & 278 \\
\hline \multicolumn{7}{|l|}{ Unit size } \\
\hline Small & $33 \%$ & $32 \%$ & $39 \%$ & $35 \%$ & 203 & 242 \\
\hline Medium & $35 \%$ & $32 \%$ & $32 \%$ & $28 \%$ & 215 & 242 \\
\hline Large & $32 \%$ & $33 \%$ & $29 \%$ & $33 \%$ & 199 & 243 \\
\hline \multicolumn{7}{|l|}{ Urbanicity $^{\dagger}$} \\
\hline Urban (1 million+) & $47 \%$ & $48 \%$ & $44 \%$ & $45 \%$ & 279 & 352 \\
\hline Other $(<1$ million $)$ & $53 \%$ & $52 \%$ & $56 \%$ & $55 \%$ & 332 & 387 \\
\hline \multicolumn{7}{|l|}{ Region } \\
\hline Midwest & $27 \%$ & $25 \%$ & $25 \%$ & $26 \%$ & 164 & 184 \\
\hline Northeast & $27 \%$ & $25 \%$ & $25 \%$ & $25 \%$ & 169 & 187 \\
\hline South & $23 \%$ & $28 \%$ & $26 \%$ & $26 \%$ & 144 & 206 \\
\hline West & $23 \%$ & $22 \%$ & $24 \%$ & $23 \%$ & 139 & 167 \\
\hline Total sample & & & & & 618 & 745 \\
\hline
\end{tabular}

${ }^{*}$ Data are weighted to account for sampling strata and nonresponse.

${ }^{\dagger}$ Data source: Area Resource File. 
These weights were constructed by multiplying the unit weight by a ratio of the number of clients (or total revenue) to mean number of clients (or mean revenue) for each unit.

A managed care arrangement was defined as the relationship between a substance abuse treatment provider and an organization that practices managed care. Some managed care arrangements are contracts that a unit have with a managed care or insurance company. For example, an OSAT unit may have a contract to provide services to members of a specific health plan for a specific period of time. Other managed care arrangements are not contractual, but may impose the same guidelines and restrictions as contractual arrangements. For example, an OSAT may have to obtain preauthorization approval from an insurer before treatment begins, even though the unit holds no formal contract with that payer. In our study, only formal, written contractual arrangements between a treatment unit and a managed care organization are considered contractual. However, respondents answered questions about their experiences with contractual and noncontractual managed care arrangements.

Treatment units were designated as having managed care if they reported that more than 10 of their OSAT clients were covered by managed care arrangements. In Wave 4,236 units met this criterion, and 310 units did so in Wave 5. Managed care was defined to encompass any contractual and noncontractual arrangements that impose treatment guidelines or restrictions which must be followed in order for OSAT units to receive payment for services provided. Directors of units with 11 or more clients covered by managed care completed the managed care section of the survey. We assumed that a minimum threshold of clients covered by managed care was necessary in order for OSAT units to exhibit particular structures, practices, or staffing arrangements. Given the wide variation in the size of OSAT units in our sample and, specifically, the many small organizations, we set the minimum threshold at 11 clients covered by managed care in the most recent fiscal year. Post hoc analysis suggests that this approach achieved essentially the same groupings of "managed care" and "nonmanaged care" OSAT units in the 1995 and 2000 samples as one using a cut-off of $10 \%$ of clients covered by managed care.

The managed care section of the survey included questions about different types of public and private managed care arrangements, the nature of the arrangements, the extent to which the unit participated in managed care, requirements of managed care organizations, and the perceived effects of managed care on OSAT. Several steps were followed to produce reliable and valid telephone survey data, including two pretests, elaborate interviewer training, extensive checks for consistency within and between sections of the survey instrument, and, when necessary, recontacts with respondents. ${ }^{16}$

This article examines (1) means and mean percentages for various measures of managed care activity for all units in the nationally representative samples at 1995 and 2000, and (2) means and mean percentages for various managed care dimensions among the treatment units that participated in managed care at each time period. In each case, the means and mean percentages are presented for relevant organizational categories, including ownership type (private for profit, private not-for-profit, public), organizational affiliation (freestanding, hospital affiliation, mental health center affiliation, other affiliation), and size (small, medium, large terciles based on number of clients per year). Chi-square and $t$ tests were used to examine differences between 1995 and 2000 in categorical and continuous variables, respectively. We used independent $t$ tests instead of Mann-Whitney tests since the assumption of normality was not critical given the large sample sizes in our study. ${ }^{17}$ Because some treatment units are present in both study years, we took a conservative approach to assessing significance and conducted independent $t$ tests using the degrees of freedom for paired $t$ tests.

\section{Results and Discussion}

In this section, data on managed care activity in the nation's outpatient drug treatment system are discussed. First, the study evaluates data regarding managed care activity among all units in the 
nationally representative samples for 1995 and 2000 (Tables 2-4).* Then, more specific information is explored about those organizations that participated in managed care (Tables 5 and 6). Except where noted, managed care includes both contractual and noncontractual arrangements.

\section{Managed care activity among all OSAT units in 1995 and 2000}

\section{Level of managed care activity}

Table 2 describes the level of managed care activity among all units in the nationally representative sample at 2 time periods, 1995 and 2000. Overall, managed care in OSAT units increased appreciably over the 5-year period. Thirty-five percent of all OSAT units were involved in managed care arrangements in 1995 compared to $47 \%$ in 2000 . The percentage of clients covered by managed care increased from $11 \%$ to $18 \%$ over the study period.

\section{Managed care and provider type}

For purposes of description, OSAT units are classified by ownership type, affiliation with other organizations, and size. Several rather striking differences were noted when examining change in managed care activity across types of OSAT units. First, private for-profit OSATs were heavily involved in managed care in 1995 and remained so in 2000 . While the percentage of for-profit units with managed care arrangements remained virtually unchanged $(47 \%-48 \%)$, the percentage of clients covered by managed care between these 2 years increased significantly $(14 \%-23 \%)$. The most pronounced growth in managed care activity occurred among private not-for-profit OSAT units. These units increased their level of involvement in managed care arrangements (40\% of units to $52 \%$ of units), and also saw a significant increase in the percentage of clients covered by managed care $(13 \%-23 \%)$.

Fewer differences were evident when comparing OSAT units across categories of organizational affiliation. Findings suggest that hospital-affiliated OSAT units continued to be actively involved in managed care, with an increase in the percentage of the clients covered by managed care between 1995 and $2000(20 \%-36 \%)$. The broadest increase in managed care activity occurred among OSAT units affiliated with mental health centers. These units increased in terms of the proportion involved in managed care $(24 \%-41 \%)$, and also in the percentage of clients covered by managed care $(8 \%-$ $15 \%)$. Freestanding OSAT units saw a significant increase in the percentage of clients covered by managed care between 1995 and $2000(6 \%-13 \%)$.

Table 2 indicates that larger OSAT units appear to have a decided advantage with respect to increasing their level of activity in managed care between 1995 and 2000 . The largest tercile of OSAT units increased by almost $100 \%$ their involvement in managed care and the percentage of clients covered by managed care. By contrast the smallest OSAT units remained relatively stable in terms of managed care involvement between 1995 and 2000. These findings suggest selective contracting on the part of managed care organizations, or that some economies may exist with respect to the ability of large organizations to take on the risk associated with providing substance abuse treatment services under managed care arrangements.

Units operating in smaller population markets ( $<1$ million) exhibited higher absolute and relative activity in managed care compared to OSAT units operating in larger population markets ( 1 million or greater). This finding applied to all 3 measures of managed care activity-percentage of units

\footnotetext{
*To assess the level of change in individual OSAT units (versus population differences), we also compared managed care activity overall and activity by organizational type for those units common to the 1995 and 2000 samples. Results for all such panel comparisons were essentially identical to those reported in Tables $2-4$ for the full samples of OSAT units. These results are available from the authors.
} 
Table 2

Managed care (MC) activity in 2 national samples of outpatient substance abuse treatment units, 1995 and 2000, by control status, unit affiliation, unit size, urbanicity, and region

\begin{tabular}{|c|c|c|c|c|c|c|c|c|}
\hline & \multicolumn{2}{|c|}{$\begin{array}{l}\% \text { of Units } \\
\text { with } \mathbf{M C}^{*}\end{array}$} & \multicolumn{2}{|c|}{$\begin{array}{c}\# \text { of MCAs, }{ }^{*}, \dagger \\
\text { Mean (SD) }\end{array}$} & \multicolumn{2}{|c|}{$\begin{array}{l}\% \text { of Clients } \\
\text { covered by } \\
\text { MC, }{ }^{\ddagger} \text { Mean\% } \\
\text { (SD) }\end{array}$} & \multicolumn{2}{|c|}{ \# of units } \\
\hline & 1995 & 2000 & 1995 & 2000 & 1995 & 2000 & 1995 & 2000 \\
\hline All units & $35 \%$ & $47 \% \S$ & $3.7(7.1)$ & $2.6(5.2)^{\S}$ & $11(22)$ & $18(29)^{\S}$ & 618 & 745 \\
\hline \multicolumn{9}{|l|}{ Control status } \\
\hline Private for profit & $47 \%$ & $48 \%$ & $6.2(9.9)$ & $2.9(5.5)^{\S}$ & $14(25)$ & $23(31)^{\|}$ & 85 & 169 \\
\hline Private not-for-profit & $40 \%$ & $52 \% \S$ & $4.8(7.1)$ & $3.0(5.7)$ & $13(23)$ & $23(32)^{\S}$ & 380 & 388 \\
\hline Public & $18 \%$ & $34 \% \S$ & $1.7(4.2)$ & $1.4(3.4)$ & $6(16)$ & $9(20)$ & 146 & 135 \\
\hline \multicolumn{9}{|l|}{ Unit affiliation } \\
\hline Freestanding & $30 \%$ & $36 \%$ & $2.8(6.3)$ & $1.8(3.9)$ & $6(17)$ & $13(27)^{\S}$ & 158 & 232 \\
\hline Hospital & $59 \%$ & $68 \%$ & $9.1(10.7)$ & $5.9(8.6)$ & $20(26)$ & $36(34)^{\S}$ & 115 & 84 \\
\hline Mental health center & $24 \%$ & $41 \% \S$ & $2.3(4.8)$ & $2.0(4.3)$ & $8(20)$ & $15(26)^{\|}$ & 138 & 106 \\
\hline Other & $33 \%$ & $47 \%^{\S}$ & $2.6(5.5)$ & $2.3(4.7)$ & $13(23)$ & $15(26)$ & 192 & 269 \\
\hline \multicolumn{9}{|l|}{ Unit size (terciles) } \\
\hline Small ( $<238$ clients $)$ & $33 \%$ & $33 \%$ & $3.6(7.5)$ & $1.6(4.6)^{\S}$ & $17(29)$ & $19(28)$ & 203 & 232 \\
\hline Medium (239-548 clients) & $38 \%$ & $49 \% \S$ & $4.0(7.3)$ & $2.5(4.7)$ & $13(23)$ & $19(29)$ & 215 & 237 \\
\hline Large (>549 clients) & $33 \%$ & $59 \% \S$ & $3.4(6.4)$ & $3.6(5.9)^{\S}$ & $10(20)$ & $19(30)^{\S}$ & 199 & 226 \\
\hline \multicolumn{9}{|l|}{ Urbanicity } \\
\hline Urban (1 million+) & $32 \%$ & $40 \%$ & $3.6(7.7)$ & $2.4(5.3)^{\| \prime}$ & $11(23)$ & $14(26)$ & 279 & 336 \\
\hline Other (<1 million) & $37 \%$ & $53 \% \S$ & $3.7(6.7)$ & $2.8(5.2)^{\|}$ & $11(21)$ & $24(32)^{\S}$ & 332 & 367 \\
\hline \multicolumn{9}{|l|}{ Region } \\
\hline Northeast & $43 \%$ & $59 \% \S$ & $3.7(7.4)$ & $3.0(4.6)$ & $19(26)$ & $22(26)$ & 164 & 170 \\
\hline Midwest & $50 \%$ & $55 \%$ & $5.8(8.5)$ & $3.5(5.9)^{\S}$ & $14(24)$ & $31(37)^{\S}$ & 169 & 177 \\
\hline South & $23 \%$ & $38 \% \S$ & $2.5(5.1)$ & $1.7(4.3)$ & $5(14)$ & $11(23)^{\prime \prime}$ & 144 & 196 \\
\hline West & $23 \%$ & $37 \% \S$ & $2.7(7.0)$ & $2.2(5.7)$ & $8(19)$ & $16(30)^{\|}$ & 139 & 163 \\
\hline
\end{tabular}

*Data are weighted by facility weights to account for sampling strata and nonresponse.

${ }^{\dagger} \mathrm{MCA}$ indicates managed care arrangements (includes contractual and noncontractual arrangements).

${ }^{\ddagger}$ Data are weighted by facility and client weights.

$\S p<.01$.

$\| p<.05$.

with managed care arrangements, number of managed care arrangements, and percentage of clients covered by managed care. Notably, the percentage of clients covered by managed care more than doubled in units operating in smaller population markets $(11 \%-24 \%)$.

Regionally, OSAT units operating in the Northeast, South, and West experienced significant growth in managed care participation between 1995 and 2000. The Northeast exhibited significant growth in the percentage of OSAT units with managed care arrangements in $2000(43 \%-59 \%)$, but was stable in the other categories of managed care activity. The Midwest, South, and West regions saw their percentage of clients covered by managed care double between 1995 and 2000. OSAT units in the South had the lowest, in average, percentage of clients covered by managed care in both 1995 and 2000 . 


\section{Table 3}

Contractual and noncontractual managed care activity in 2 national samples of outpatient substance abuse treatment units, 1995 and 2000, by control status, unit affiliation, unit size, urbanicity, and region*

\begin{tabular}{|c|c|c|c|c|c|c|c|c|}
\hline & \multicolumn{2}{|c|}{$\begin{array}{l}\text { Total \# of MCAs, }{ }^{\dagger} \\
\text { Mean (SD) }\end{array}$} & \multicolumn{2}{|c|}{$\begin{array}{c}\text { \# of Contractual } \\
\text { MCAs, Mean (SD) }\end{array}$} & \multicolumn{2}{|c|}{$\begin{array}{l}\text { \# of Noncontractual } \\
\text { MCAs, Mean (SD) }\end{array}$} & \multicolumn{2}{|c|}{ \# of units } \\
\hline & 1995 & 2000 & 1995 & 2000 & 1995 & 2000 & 1995 & 2000 \\
\hline All units & $3.7(7.1)$ & $2.6(5.2)^{\ddagger}$ & $2.7(6.4)$ & $1.7(3.9)^{\ddagger}$ & $0.9(2.8)$ & $0.9(2.9)$ & 618 & 745 \\
\hline \multicolumn{9}{|l|}{ Control status } \\
\hline Private for profit & $6.2(9.9)$ & $2.9(5.5)^{\ddagger}$ & $4.4(8.6)$ & $2.2(3.8)^{\S}$ & $1.1(2.7)$ & $0.9(2.5)$ & 79 & 169 \\
\hline Private not-for-profit & $4.8(7.1)$ & $3.0(5.7)$ & $3.0(6.0)$ & $2.1(4.5)^{\S}$ & $1.1(3.4)$ & $1.0(3.3)$ & 345 & 388 \\
\hline Public & $7(4.2)$ & $1.4(3.4)$ & $1.5(0.7)$ & $0.7(1.8)$ & $0.4(1.7)$ & $0.7(2.4)$ & 133 & 135 \\
\hline \multicolumn{9}{|l|}{ Unit affiliation } \\
\hline Freestanding & $2.8(6.3)$ & $1.8(3.9)$ & $1.8(4.9)$ & $1.3(3.0)$ & $1.1(3.1)$ & $0.5(1.7)^{\S}$ & 147 & 232 \\
\hline Hospital & $9.1(10.7)$ & $5.9(8.6)$ & $7.9(10.3)$ & $5.0(8.2)$ & $1.7(4.1)$ & $0.9(2.2)$ & 104 & 84 \\
\hline Mental & $2.3(4.8)$ & $2.0(4.3)$ & $2.2(6.4)$ & $0.8(1.9)^{\S}$ & $0.4(1.9)$ & $1.1(3.0)$ & 122 & 106 \\
\hline Other & $6(5.5)$ & 2.3 & $1.4(3.2)$ & $1.3(2.5)$ & 0.5( & $1.0(3.4)$ & 181 & 269 \\
\hline \multicolumn{9}{|l|}{ Unit size (terciles) } \\
\hline Small (<238 clients) & $3.6(7.5)$ & $1.6(4.6)^{\ddagger}$ & $2.3(6.6)$ & $1.3(4.2)$ & $0.9(2.7)$ & $0.3(1.0)^{\ddagger}$ & 185 & 232 \\
\hline Medium (239-548 clients) & $4.0(7.3)$ & $2.5(4.7)$ & $3.0(6.7)$ & $1.4(2.7)^{\ddagger}$ & $1.1(3.4)$ & $1.1(3.2)$ & 190 & 237 \\
\hline Large $(>54$ & $3.4(6.4)$ & $3.6(5.9)^{\ddagger}$ & $2.8(2.4)$ & $2.4(4.4)$ & $0.7(2.3)$ & $1.2(3.4)$ & 183 & 226 \\
\hline \multicolumn{9}{|l|}{ Urbanicity } \\
\hline Urban (1 million + ) & $3.6(7.7)$ & $2.4(5.3)^{\S}$ & $2.6(7.1)$ & & $0.6(1.9)$ & $0.7(2.3)$ & 279 & 336 \\
\hline Other $(<1$ & $3.7(6.7)$ & $2.8(5.2)^{\S}$ & $2.9(5.9)$ & $1.8(3.7)^{\ddagger}$ & $1.1(3.4)$ & $1.1(3.2)$ & 332 & 367 \\
\hline \multicolumn{9}{|l|}{ Region } \\
\hline Northeast & $3.7(7.4)$ & $3.0(4.6)$ & $2.5(6.0)$ & $2.1(3.9)$ & $0.7(2.1)$ & $0.7(1.7)$ & 164 & 170 \\
\hline Midwest & $5.8(8.5)$ & $3.5(5.9)^{\ddagger}$ & $4.6(8.3)$ & $2.0(3.2)^{\dagger}$ & $1.7(4.4)$ & $1.5(4.2)$ & 169 & 177 \\
\hline South & $2.5(5.1)$ & $1.7(4.3)$ & $2.5(6.5)$ & $1.2(3.7)^{\S}$ & $0.8(2.4)$ & $0.7(2.5)$ & 144 & 196 \\
\hline West & $2.7(7.0)$ & $2.2(5.7)$ & $1.1(3.1)$ & $1.6(4.9)$ & $0.2(0.9)$ & $0.8(2.6)^{\S}$ & 139 & 163 \\
\hline
\end{tabular}

${ }^{*}$ Data are weighted to account for sampling strata and nonresponse.

${ }^{\dagger} \mathrm{MCA}$ indicates managed care arrangements.

${ }^{\dagger} p<.01$.

$\S^{\S} p<.05$.

Because our definition of managed care encompassed both contractual and noncontractual arrangements, we also explored whether differences in the findings reported above obtain for each of these 2 categories of managed care arrangements. Results (Table 3 ) indicate that contractual managed are arrangements are both more common and, in general, experienced more consolidation between 1995 and 2000 relative to noncontractual, managed care arrangements.

\section{Types of public and private managed care arrangements}

Table 4 presents data on changes in the types of public and private managed care arrangements found among all OSAT units. Here, public managed behavioral care programs (eg, those sponsored by federal, state, and local governments) are distinguished from private managed behavioral care programs (eg, those offered by various private for-profit and not-for-profit firms). Table 4 indicates that the percentage of units participating in Medicaid increased between 1995 and $2000(17 \%-26 \%)$. Nationally, the percentage of units with all other public managed care arrangements in force remained stable at around $14 \%$ in both 1995 and 2000. 


\section{Table 4}

Private and public managed care arrangements in 2 national samples of outpatient substance abuse treatment (OSAT) units, 1995 and $2000^{*, \dagger}$

\begin{tabular}{|c|c|c|}
\hline & $\begin{array}{c}1995 \\
(N=593)\end{array}$ & $\begin{array}{c}2000 \\
(N=619)\end{array}$ \\
\hline \multicolumn{3}{|c|}{ Percentage of OSAT units with private managed care arrangements ${ }^{*, \ddagger}$} \\
\hline $\mathrm{HMO}^{\S}$ & 31 & 29 \\
\hline $\mathrm{PPO}$ or $\mathrm{EPO}^{\S}$ & 27 & $19^{\|}$ \\
\hline $\mathrm{EAP}^{\S}$ & 25 & 12 \\
\hline Direct contracts with self-insured firms & 13 & $8^{\#}$ \\
\hline Insurance companies & 28 & 27 \\
\hline Other private organizations & 3 & 3 \\
\hline \multicolumn{3}{|c|}{ Percentage of OSAT units with public managed care arrangements* } \\
\hline Medicaid & 17 & $26^{\#}$ \\
\hline Other government organization & 13 & 15 \\
\hline State substance abuse agency & 6 & 7 \\
\hline Other state agency & 2 & 2 \\
\hline Courts, prisons & 5 & 4 \\
\hline Other local government & 5 & 3 \\
\hline Medicare & 5 & 4 \\
\hline Other federal programs & 2 & 2 \\
\hline
\end{tabular}

${ }^{*}$ Includes both contractual and noncontractual arrangements.

${ }^{\dagger}$ Data are weighted to account for sampling strata and nonresponse.

${ }^{\ddagger}$ Because units may have managed care arrangements with more than one of the subcategories, numbers do not sum to $100 \%$.

${ }^{\S} \mathrm{HMO}$ indicates health maintenance organization; PPO or EPO, preferred provider organization or exclusive provider organization; EAP, employee assistance program.

$\| p<.05$.

$\pi_{p}<.001$.

${ }^{\#} p<.01$.

Although OSAT units overall report having more private managed care arrangements than public ones, private managed care arrangements experienced no growth between 1995 and 2000. Specifically, the percentage of units indicating that they had managed care arrangements with preferred provider organizations (PPOs) or exclusive provider organizations (EPOs), employee assistance programs (EAPs), and direct contracts with firms declined between 1995 and 2000.

\section{Managed care activity among units with managed care in 1995 and 2000}

\section{Public and private managed care activity}

Tables 5 and 6 display changes in public and private behavioral managed care activity for OSAT units that have more than 10 clients covered by managed care arrangements. As shown in the tables, there is variation in public and private managed care activity across all types of OSAT providers.

Among all units involved in managed care, there appears to be relatively little change between 1995 and 2000 in the level of public managed care activity in OSAT units (Table 5). Overall, the number of public managed care arrangements declined from 1.4 to 1.0 , but there is no significant 


\section{Table 5}

Public managed care (MC) activity in treatment units with MC, 1995 and 2000, by control status, unit affiliation, unit size, urbanicity, and region

\begin{tabular}{|c|c|c|c|c|c|c|c|c|}
\hline & \multicolumn{2}{|c|}{$\begin{array}{l}\text { \# of Public } \\
\text { MCAs, } \\
\text { Mean (SD) }\end{array}$} & \multicolumn{2}{|c|}{$\begin{array}{c}\% \text { of Clients in } \\
\text { public MC }{ }^{\dagger} \\
\text { Mean } \%(\text { SD) }\end{array}$} & \multicolumn{2}{|c|}{$\begin{array}{c}\% \text { of Public } \\
\text { MC revenue, } \ddagger \\
\text { Mean \% (SD) }\end{array}$} & \multicolumn{2}{|c|}{$N$} \\
\hline & 1995 & 2000 & 1995 & 2000 & 1995 & 2000 & 1995 & 2000 \\
\hline MC units & $1.4(3.8)$ & $1.0(1.7)^{\S}$ & $23.5(32.5)$ & $18.9(26.8)$ & $21.3(30.0)$ & $19.9(30.1)$ & 236 & 310 \\
\hline \multicolumn{9}{|l|}{ Control status } \\
\hline Private for profit & $1.6(3.8)$ & $0.6(0.91)$ & $19.6(34.5)$ & $9.5(19.7)$ & $11.2(26.6)$ & $10.5(26.6)$ & 44 & 68 \\
\hline Private not-for-profit & $1.4(4.2)$ & $1.0(1.3)$ & $23.8(32.2)$ & $23.1(28.8)$ & $23.8(26.4)$ & $26.4(33.5)$ & 156 & 184 \\
\hline Public & $1.2(1.7)$ & $1.3(3.1)$ & $26.8(30.7)$ & $12.4(20.9)^{\S}$ & $21.1(27.6)$ & $8.9(14.5)^{\S}$ & 36 & 42 \\
\hline \multicolumn{9}{|l|}{ Unit affiliation } \\
\hline Freestanding & $1.2(2.6)$ & $0.7(1.1)$ & $23.8(32.9)$ & $18.6(28.9)$ & $22.0(28.0)$ & $24.0(34.4)$ & 53 & 82 \\
\hline Hospital & $1.5(2.6)$ & $0.9(0.90)$ & $14.8(22.7)$ & $14.9(19.6)$ & $13.5(21.7)$ & $10.7(20.1)$ & 66 & 50 \\
\hline Mental health center & $1.4(2.1)$ & $0.9(0.65)$ & $38.6(40.4)$ & $20.9(27.3)^{\S}$ & $34.1(39.8)$ & $14.0(22.4)^{8}$ & 45 & 43 \\
\hline Other & $1.5(3.2)$ & $1.2(2.6)$ & $21.8(30.1)$ & $15.6(24.2)$ & $20.6(21.5)$ & $21.5(31.6)$ & 64 & 121 \\
\hline \multicolumn{9}{|l|}{ Unit size (terciles) } \\
\hline Small (<238 clients) & $1.7(3.3)$ & $0.8(0.96)^{8}$ & $23.4(30.6)$ & $22.1(31.9)$ & $27.6(37.8)$ & $23.5(33.6)$ & 72 & 74 \\
\hline Medium (239-548 clients) & $0.9(1.8)$ & $0.7(0.61)$ & $14.2(23.6)$ & $20.7(28.9)$ & $16.5(24.3)$ & $21.1(30.6)$ & 84 & 106 \\
\hline Large $(>549$ clients $)$ & $1.7(2.6)$ & $1.4(2.5)$ & $26.6(34.5)$ & $18.4(26.1)^{8}$ & $22.8(30.7)$ & $18.8(29.3)$ & 80 & 122 \\
\hline \multicolumn{9}{|l|}{ Urbanicity } \\
\hline Urban (1 million+) & $1.4(2.7)$ & $0.9(0.8)^{\hat{9}}$ & $21.9(32.9)$ & $17.7(23.8)$ & $23.4(32.9)$ & $17.2(29.5)$ & 98 & 129 \\
\hline Other $(<1$ million $)$ & $1.4(2.7)$ & $1.0(2.1)$ & $24.5(32.3)$ & $19.6(27.3)$ & $19.1(27.0)$ & $21.9(30.5)$ & 137 & 181 \\
\hline \multicolumn{9}{|l|}{ Region } \\
\hline Northeast & $1.3(3.1)$ & $0.8(0.8)$ & $26.4(33.5)$ & $19.4(26.8)$ & $23.5(26.3)$ & $19.0(31.2)$ & 76 & 100 \\
\hline Midwest & $1.2(2.0)$ & $1.0(1.4)$ & $10.8(23.1)$ & $20.7(29.3)^{8}$ & $17.3(23.9)$ & $23.9(31.2)$ & 84 & 91 \\
\hline South & $1.9(2.8)$ & $1.2(3.0)$ & $26.9(34.4)$ & $14.5(24.5)^{3}$ & $8.9(22.3)$ & $13.7(24.5)$ & 38 & 58 \\
\hline West & $1.3(3.1)$ & $1.0(1.2)$ & $37.5(35.6)$ & $22.1(26.4)^{\S}$ & $34.6(39.6)$ & $23.3(31.7)^{8}$ & 38 & 62 \\
\hline
\end{tabular}

*MCA indicates managed care arrangement (includes contractual and noncontractual arrangements). Data are weighted to account for sampling and nonresponse.

${ }^{\dagger}$ Data are weighted by facility and client weights.

${ }^{\ddagger}$ Data are weighted by facility and revenue weights.

$\S_{p}<.05$.

change in the percentage of clients or the percentage of revenues covered by public managed care between 1995 and 2000.

However, a few significant changes are observed when looking at specific types of OSAT providers. First, public managed care activity declined among public treatment organizations between 1.995 and 2000. Specifically, the percentage of clients covered by public managed care arrangements decreased from $27 \%$ to $12 \%$ and the percent of unit revenues from public managed care decreased from $21 \%$ to $9 \%$ for these organizations. OSAT units affiliated with mental health centers also saw a significant decrease between 1995 and 2000 in both the percentage of clients covered by public managed care $(39 \%-21 \%)$ and in the percentage of revenue from public managed care arrangements $(34 \%-14 \%)$.

Few changes are noted by either size of market population or region. The exceptions of note include an increase in the percentage of clients covered by public managed care in OSAT units in the Midwest, and a comparable decline in percentage of public managed care clients for units operating in the South. Units in the West saw a decrease in the percentage of clients covered by public managed care and also in the percentage of revenue from public managed care arrangements.

Overall, the level of private managed care activity for units participating in managed care declined over the 1995-2000 period as measured by the number of private managed care arrangements and the 


\section{Table 6}

Private managed care (MC) activity in treatment units with MC, 1995 and 2000, by control status, unit affiliation, unit size, urbanicity, and region

\begin{tabular}{|c|c|c|c|c|c|c|c|c|}
\hline & \multicolumn{2}{|c|}{$\begin{array}{c}\text { \# of Private } \\
\text { MCAs, }{ }^{*} \\
\text { Mean (SD) }\end{array}$} & \multicolumn{2}{|c|}{$\begin{array}{l}\% \text { of Clients in } \\
\text { private MC }{ }^{\dagger} \\
\text { Mean \% (SD) }\end{array}$} & \multicolumn{2}{|c|}{$\begin{array}{l}\text { \% of Private } \\
\text { MC revenue, } \\
\text { Mean \% (SD) }\end{array}$} & \multicolumn{2}{|c|}{$N$} \\
\hline & 1995 & 2000 & 1995 & 2000 & 1995 & 2000 & 1995 & 2000 \\
\hline MC units & $7.4(8.2)$ & $4.7(6.2)^{\S}$ & $24.2(27.8)$ & $21.4(25.8)$ & $25.9(26.9)$ & $19.5(28.0)^{\Upsilon}$ & 236 & 310 \\
\hline \multicolumn{9}{|l|}{ Control status } \\
\hline Private for profit & $10.5(10.4)$ & $5.4(6.5)^{\|}$ & $36.0(30.1)$ & $37.0(34.0)$ & $48.2(34.0)$ & $39.2(30.9)$ & 44 & 68 \\
\hline Private not-for-profit & $6.5(11.9)$ & $5.1(6.6)$ & $23.6(28.1)$ & $21.3(24.4)$ & $25.0(30.8)$ & $17.9(27.2)^{9}$ & 156 & 184 \\
\hline Public & $6.5(5.8)$ & $3.0(3.7)^{l l}$ & $13.0(15.8)$ & $10.2(14.7)$ & $13.5(17.2)$ & $5.8(9.3)^{\top}$ & 36 & 42 \\
\hline \multicolumn{9}{|l|}{ Unit affiliation } \\
\hline Freestanding & $7.1(7.8)$ & $4.2(5.0)^{9}$ & $24.6(30.1)$ & $24.5(24.1)$ & $25.9(31.4)$ & $20.5(22.7)$ & 53 & 82 \\
\hline Hospital & $11.2(10.9)$ & $8.1(9.1)$ & $38.2(30.3)$ & $34.2(33.9)$ & $43.2(31.1)$ & $32.2(35.7)$ & 66 & 50 \\
\hline Mental health center & $5.1(3.9)$ & $4.0(5.6)$ & $16.8(29.9)$ & $10.8(15.4)$ & $20.2(31.7)$ & $6.1(12.3)^{\top}$ & 45 & 43 \\
\hline Other & $5.6(6.3)$ & $3.8(5.0)^{9}$ & $19.0(17.9)$ & $19.7(24.6)$ & $17.8(25.8)$ & $13.9(24.5)$ & 64 & 121 \\
\hline \multicolumn{9}{|l|}{ Unit size (terciles) } \\
\hline Small ( $<238$ clients) & $8.1(8.9)$ & $4.2(7.0)^{\|}$ & $30.3(30.8)$ & $23.5(26.3)$ & $24.5(28.9)$ & $15.6(28.4)$ & 72 & 74 \\
\hline Medium (239-548 clients) & $7.1(4.5)$ & $4.5(5.6)^{9}$ & $32.1(34.5)$ & $18.9(24.8)^{9}$ & $30.5(33.9)$ & $23.9(28.7)$ & 84 & 106 \\
\hline Large (>549 clients) & $6.9(7.6)$ & $5.1(5.9)$ & $20.8(24.0)$ & $21.8(25.9)$ & $25.9(30.6)$ & $15.1(24.6)^{\Upsilon}$ & 80 & 122 \\
\hline \multicolumn{9}{|l|}{ Urbanicity } \\
\hline Urban (1 million+) & $8.4(9.7)$ & $5.1(7.0)^{\|}$ & $31.2(30.3)$ & $22.9(30.4)$ & $36.3(35.9)$ & $19.2(27.4)^{4}$ & 97 & 124 \\
\hline Other ( $<1$ million) & $6.8(7.0)$ & $4.5(5.6)^{\|}$ & $20.0(25.2)$ & $20.5(22.4)$ & $19.5(24.5)$ & $17.0(26.0)$ & 137 & 172 \\
\hline \multicolumn{9}{|l|}{ Region } \\
\hline Northeast & $5.8(7.4)$ & $4.4(5.0)$ & $19.2(20.0)$ & $18.3(20.3)$ & $18.0(23.1)$ & $15.1(20.7)$ & 76 & 98 \\
\hline Midwest & $8.4(8.9)$ & $5.6(6.4)^{4}$ & $30.2(33.1)$ & $27.0(30.9)$ & $37.2(34.9)$ & $27.2(32.6)$ & 83 & 88 \\
\hline South & $7.3(5.5)$ & $3.7(5.6)^{\| \prime}$ & $18.8(21.6)$ & $18.0(24.4)$ & $24.4(30.2)$ & $17.8(31.1)$ & 38 & 54 \\
\hline West & $8.6(10.3)$ & $5.2(7.8)$ & $29.1(32.9)$ & $24.0(27.9)$ & $30.4(35.7)$ & $11.7(19.5)$ & 37 & 57 \\
\hline
\end{tabular}

* MCA indicates managed care arrangement (includes contractual and noncontractual arrangements). Data are weighted to account for sampling and nonresponse.

${ }^{\dagger}$ Data are weighted by facility and client weights.

† Data are weighted by facility and revenue weights.

${ }^{\S} p<.001$.

$\| p<.01$.

$\Upsilon_{p}<.05$.

percentage of revenue from private managed care arrangements (Table 6). Specifically, the number of managed care arrangements dropped from 7.4 to 4.7 , and the percentage of revenues covered by private managed care decreased from $26 \%$ to $20 \%$ from 1995 to 2000.

These declines are consistent across several categories of OSAT units. Despite a decline in the number of private managed care arrangements, private for-profit OSAT units continued to be dominant participants in the private managed care arena, whereas private not-for-profit units and public units declined precipitously in terms of the percentage of revenues covered by private managed care arrangements. Public units also saw a decrease in the number of private managed care arrangements. These particular patterns of consolidation may be a consequence of increased selective contracting on the part of managed care organizations.

OSAT units affiliated with mental health centers, larger units, and urban units also experienced a decline in revenue from private managed care arrangements. Medium-sized units experienced a significant decline in the private managed care clients. 


\section{Implications for Behavioral Health Services}

This national study reveals that, overall, managed behavioral care increased between 1995 and 2000 among all OSAT units nationally. However, this increase is not uniform across all types of providers, or all types of managed care programs. This may be the function of unequal penetration of managed care in certain markets and/or selective contracting by managed care firms that seek out providers with certain characteristics. Several specific findings are indicative of these selective changes in managed care activity among OSAT units and have implications for behavioral health services, as discussed below.

First, there has been a modest decline in private managed behavioral care activity, particularly in terms of number of managed care arrangements and percentage of revenue covered by private managed care. This decline may reflect a shaking out of the managed care market in which many private managed care organizations are succumbing to competitive pressures in the marketplace. The downward trend for private managed care contrasts with the increase in public managed care activity among OSAT providers nationally. In particular, Medicaid managed care has increased over the study period, while all types of private managed care decreased. One possibility is that the increase in public managed care activity and the decline in the private managed care sector may ultimately contribute to a 2-tiered substance abuse treatment system. These data, however, suggest that most OSAT units treat both publicly and privately insured patients. In fact, while there are some units that may be considered "niche" or "specialized" providers, the vast majority of OSAT units are dealing with both private and public managed behavioral care organizations. To the extent that this creates confusion and conflicting incentives for providers at the point of treatment, policymakers must concern themselves with how potentially conflicting requirements have an impact on the type and quality of care provided to substance abuse clients. On the other hand, the fact that most OSAT units treat clients under both public and private managed care arrangements suggests that concerns about a 2-tiered system of care and differential treatment of public and privately funded clients may be unfounded.

These data also suggest that OSAT units that do participate in managed care are involved in fewer managed care arrangements than they were 5 years ago. Although there is little, if any, single-source managed care contracting in the substance abuse arena, the number of contracts held by OSAT organizations is clearly in decline. This may be due to at least 2 factors. First, the market itself may be consolidating such that fewer managed care firms are actively seeking providers for their networks. Second, from the perspective of the OSAT unit itself, dealing with fewer managed care organizations is far easier administratively than managing relationships with many firms. Thus, OSAT units may prefer to move toward fewer contracts rather than multiple contracts. The fact that these organizations may have to deal with fewer conflicting sets of administrative requirements may ultimately have the effect of reducing overall substance abuse treatment costs.

A third consistent finding emerging from this study is that managed care activity has increased among units affiliated with other types of health and social service organizations, such as hospitals and mental health care centers. This may reflect 2 factors. The first is the increasing recognition that substance abuse is a multifaceted problem, involving social, economic, and physical health. The ability to coordinate and link substance abuse treatment to other forms of care may be attractive to managed care firms. A second explanation is that linkages with other providers may simply provide the size necessary to assume the risks associated with managed care contracts. The finding that managed care growth has been most pronounced among larger OSAT units and is virtually nonexistent among smaller providers supports this latter explanation. A third possibility is that the preference for larger, hospital and mental health center affiliated providers may reflect a desire on the part of managed care organizations to purchase a more comprehensive array of services from the same provider. An important policy question raised by this pattern of findings is the ability of smaller, freestanding units to secure managed care arrangements of any type, and the consequences for service 
provision and access by those requiring care from such providers. If managed care becomes a more important source of funding in behavioral health care, the future of these organizations and their clients may be at risk.

Finally, although private for-profit firms still command a substantial share of the managed care market among OSAT units, the relative balance among the categories of OSAT units appears to be more equal in 2000 than it was in 1995. Such balance might bode well for access to care among different categories of clients and provide alternatives to cost-effective care for those requiring substance abuse treatment service. The analyses presented in this article are largely descriptive and assess managed care activity in OSAT units at a general level. To understand the consequences of the type of activity reported in this article, it will be necessary to identify with greater specificity how behavioral managed care affects the provision of services to substance abuse clients. Some have argued, for example, that behavioral managed care organizations do not engage in risk transfer to providers of substance abuse services. ${ }^{18}$ Under this scenario little difference would be expected in provider behavior vis-a-vis other forms of insurance. On the other hand, other research has reported that many behavior managed care firms impose very specific requirements for treatment access, and methods, and that such requirements vary greatly across managed care organizations. ${ }^{19,20}$ Understanding how these different expressions of behavioral managed care may ultimately affect the quality, access and cost of treatment provided in OSAT units should represent a high priority for policymakers and researchers.

Of course, the trends in managed care reported in this article derive their greatest importance from their potential effects on access to care and utilization of substance abuse treatment services by clients requiring such services. For example, as managed care expands into the public sector, policymakers must attend to the possibility that cost containment may reduce the range and type of services available to vulnerable populations. Inpatient care, ancillary services, and treatment follow-up are particularly vulnerable in this regard. Alternatively, rationalizing care through public managed care may provide greater access to care than the "hit or miss" approaches used in the past. Linking the change patterns reported here to access, utilization, and treatment practices will also require greater attention to micro-level organizational analysis to understand how OSAT units respond to managed care imperatives. For example, a recent study reported that treatment units with both relatively low and relatively high managed care penetration were more likely to support access to care; these units provided care to higher percentages of clients unable to pay and were less likely to shorten treatment because of client inability to pay. ${ }^{21}$ Treatment units with midrange managed care penetration were least likely to support access to care. Further study might explore whether OSAT units and the providers that work in these units can distinguish between clients covered by private insurance versus private managed care, or Medicaid managed care from private managed care. Multilevel studies that link broad trends in managed care to adaptive responses on the part of individual treatment units is a logical next step in this line of inquiry.

\section{Acknowledgments}

Grants 5RO1-DA03272 and 5RO1-DA087231 from the National Institute of Drug Abuse (NIDA) supported this research. The views expressed here are the authors' and not necessarily those of NIDA. The authors thank Xihong Lin and Thomas A. D'Aunno for their counsel and recommendations.

\section{References}

1. Open Minds. Yearbook of Managed Behavioral Health Market Share in the United States, 2000-2001. Gettysburg, Pa: Open Minds; 2000.

2. Substance Abuse and Mental Health Services Administration. State Profiles, 1999, on Public Sector Managed Behavioral Health Care. Washington, DC: US Govemment Printing Office; 2000. DHHS Publication No. (SMA)00-3432.

3. Findlay S. Managed behavioral care in 1999: an industry at the crossroads. Health Affairs. 1999;18(5):116-124. 
4. Sturm R. Managed care risk contracts and substance abuse treatment. Inquiry. 2000;37(2):219-225.

5. Alexander JA, Lemak CH. The effects of managed care on administrative burden in outpatient substance abuse treatment facilities. Medical Care. 1997;35(10): 1060-1068.

6. Liu X, Sturm R, Cuffel B. The impact of preauthorization on outpatient utilization in managed behavioral health plans. Medical Care Research and Review. 2000;57(2):182-195.

7. Stein B, Orlando M, Sturm R. The effect of copayments on drug and alcohol treatment following inpatient detoxification under managed care. Psychiatric Services. 2000;51(2):195-198.

8. Sturm R. Cost and quality trends under managed care: is there a learning curve in behavioral health carve-out plans? Journal of Health Economics. 1999;18:593-604.

9. Substance Abuse and Mental Health Services Administration. Overview of the National Drug and Alcoholism Treatment Unit Survey (NDATUS): 1992 and 1980-1992. Rockville, Md: Substance Abuse and Mental Health Services Administration; 1995. Advance Report Number 9.

10. Zarkin GA, Galinis DN, French MT, et al. Financing strategies for drug abuse treatment programs. Journal of Substance Abuse Treatment 1995; 12:385-399.

11. Essock SM, Goldman HH. States embrace of managed mental health care. Health Affairs. 1995;14:34-44.

12. Garnick DW, Hendricks AM, Dulski JD, et al. Characteristics of private-sector managed care for mental health and substance abuse treatment. Hospital and Community Psychiatry. 1994;45:1201-1205.

13. Price RH, Burke AC, D'Aunno TA, et al. Outpatient drug abuse treatment services, 1988: results of a national study. In: Pickens RW, Lukefeld CG, Schuster CR, eds. Improving Drug Abuse Treatment. Rockville, Md: National Institute on Drug Abuse; 1991:63-92.

14. Heeringa SG. Outpatient Drug Abuse Treatment Studies: Technical Documentation. Ann Arbor, Mich: Institute for Social Research, Survey Design and Analysis Unit, University of Michigan; 1996.

15. Adams TA, Heeringa SG. Outpatient Substance Abuse Treatment System Surveys (OSATSS): Technical Documentation for OSATSS-5, 1999-2000. Ann Arbor, Mich: Institute for Social Research, Survey Design and Analysis Unit, University of Michigan; 2001

16. Groves RM, Biemer PP, Lyberg LE, et al, eds. Telephone Survey Methodology. New York: John Wiley \& Sons; 1988.

17. Hays WL. Statistics. 5th ed. Fort Worth, Tex: Harcourt Brace College Publishers; 1994.

18. Garnick D, Horgan C, Hodgkin D, et al. Risk transfer and accountability in managed care organizations carve out contracts. Psychiatric Services. 2001;52(11):1502-1509.

19. Sosin MR, D'Aunno TA. The organization of substance abuse managed care. Recent Developments in Alcoholism. 2001; 15:27-49.

20. Lemak CH, Alexander JA. Managed care and outpatient substance abuse treatment intensity. Journal of Behavioral Health Services \& Research. $2001 ; 28(1): 12-29$.

21. Alexander JA, Nahra TA, Wheeler JR. Managed care and access to substance abuse treatment services. Journal of Behavioral Health Services \& Research. 2003;30(2):161-165.

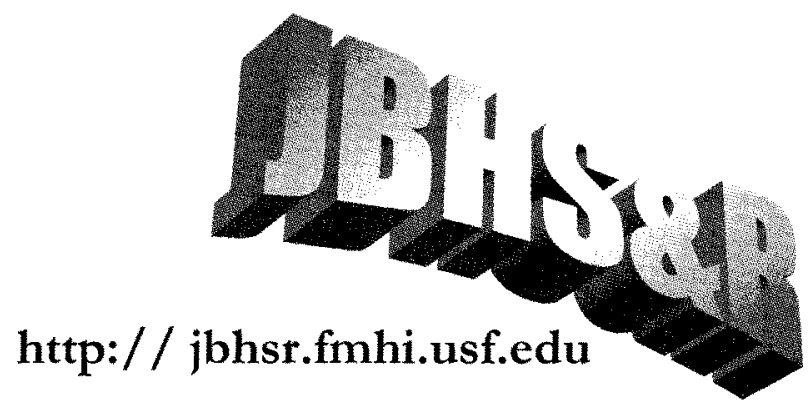

